\title{
In-process measurements of material removal in fluid jet polishing
}

\author{
Hedser van $\operatorname{Brug}^{a}$, Mietta Groeneveld $^{b}$, Silvia M. Booij ${ }^{b}$ and Joseph J.M. Braat ${ }^{b}$ \\ ${ }^{a}$ TNO TPD, Stieltjesweg 1, 2628 CK Delft, the Netherlands \\ ${ }^{b}$ Delft University of Technology, Optics Research Group \\ Lorentzweg 1, 2628 CJ Delft, the Netherlands
}

\begin{abstract}
A prototype of a system for in-process monitoring of material removal in fluid jet polishing (FJP) is presented. The measurements make use of temporal phase unwrapping (TPU) allowing for a large working range. The measurement system will be discussed, with all problems that had to be overcome like water on the surface and vibrations, as well as the FJP system. The basics behind TPU will be presented and the first results will be shown. Finally, the capabilities of the system will be discussed. The presented system enables the in-process monitoring of the footprint as obtained by the FJP technique and measurement of the material removal rate.
\end{abstract}

Keywords: In-process, Temporal phase unwrapping, Interferometry, Optical fabrication, Polishing

\section{INTRODUCTION}

During the fabrication of optical components, being it lenses, mirrors or prisms, there are moments at which it is important to know what the status of the surface at that time is. Often the shape is important but also the surface quality is an issue. For the case that the end shape of the surface is of importance the starting shape has to be known, as well as the footprint of the machining tool and the material removal rate of the tool. Knowing all this should in principle be enough to arrive at perfect final surfaces. In reality this is never the case. Either the footprint is found to be non stationary, or the material removal changes during the process, etc. To arrive at a good final surface it is common practice to remove at regular basis the object from the machine, measure its surface shape and determine the places where material has to be removed, and replace the object on the machine for further processing. This is a very time consuming process due to its iterative nature.

Apart from being time consuming this approach is also error prone. Due to instabilities in the process it often happens that more material is being removed than required. Since replacing of that material is not an option, all other areas have to be machined as well in order to arrive at the desired end shape.

This paper deals with a tool to monitor the material removal during processing. Using this system very accurate machining footprints can be obtained allowing for very accurate surface shaping. Also the material removal rate can be monitored under very different circumstances.

Although surface roughness has been mentioned, this paper will not discuss the tool, iTIRM, that was developed by us. iTIRM, intensity-detecting total internal reflection microscopy, is a simple tool where the specular reflected intensity is monitored. Details on iTIRM can be found in literature. ${ }^{1}$

In the following sections the fluid jet system will be described, followed by an introduction in temporal phase unwrapping. After that the used setup will be presented together with result obtained by it. Finally the results will be discussed and some conclusions will be drawn from this research.

Further author information:

Hedser van Brug: E-mail: brug@tpd.tno.nl, Telephone: +31 152692489 


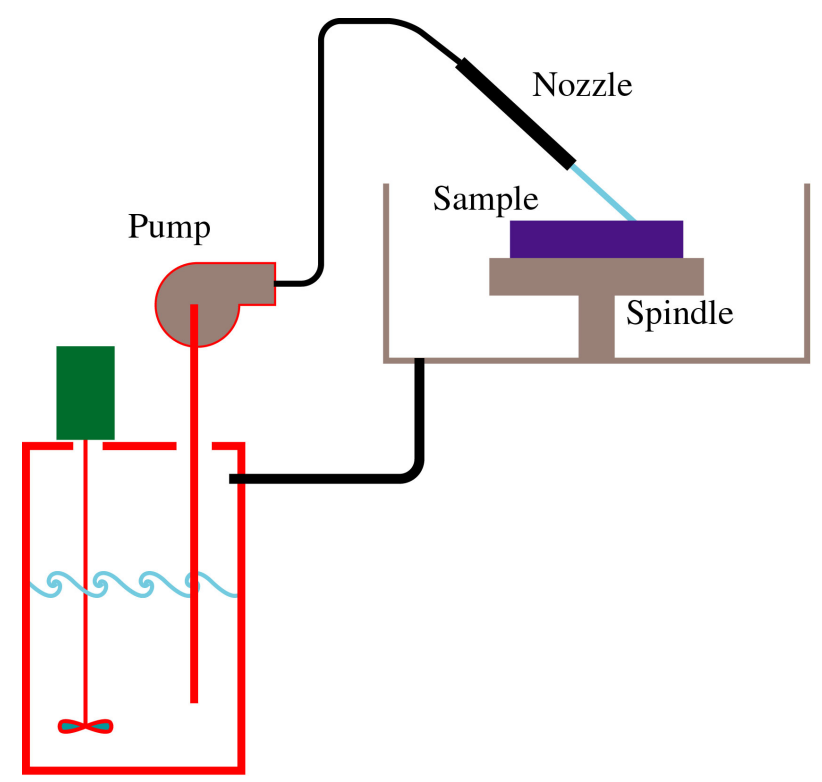

Figure 1. Layout of the FJP system.

\section{FLUID JET POLISHING}

Fluid jet polishing, or short FJP, is a novel method to shape and polish all kinds of surfaces. ${ }^{2}$ An FJP system consists of a container in which abrasive particles, e.g. $\mathrm{SiC}$ or $\mathrm{Al}_{2} \mathrm{O}_{3}$, are mixed with the liquid, in our case water. This mixture, the slurry, is pumped through a nozzle. The jet that is formed by the nozzle impinges on the surface being processed, see Fig. 1. The slurry jet characteristics depend among others on the nozzle shape, which on their turn influence the profile of the spot being generated, the removal rate and the finally obtainable surface roughness.

In Fig. 1 the slurry container, the pump and the nozzle are shown. It is found that the FJP process is insensitive to the offset between the nozzle and the workpiece. Benefits are that it is a relative cheap system, that it can be used both for shaping and finishing of optical components, and that it removes debris from the surface. The slurry can be reused and the water acts as coolant for the workpiece. During polishing, material is removed while the surface roughness remains unchanged and during shaping both material is removed and surface roughness is improved.

The shape of the hollow created by the FJP process, the spot or footprint, depends on the angle of incidence of the jet to the surface and on the nozzle geometry. This has been discussed previously. ${ }^{3}$

The removal rate depends on, as mentioned before the nozzle, the applied pressure (typically between 1 and 10 bar), the type of abrasive particles, the type of surface being processed, and on the concentration of the abrasives in the slurry (typically 1 - 15 wt.\% of \#800 SiC). The removal rate can be high, several $\mu m$ per minute or very low, several $\mathrm{nm}$ per minute, depending on the concentration and abrasives type. The footprint of the FJP system has an area of about $7 \mathrm{~mm}^{2}$, depending on the nozzle geometry and on the offset between nozzle and surface. The removal rate was found to hardly depend on the standoff distance of the nozzle with respect to the surface.

\section{TEMPORAL PHASE UNWRAPPING}

In this section the basics behind temporal phase unwrapping will be discussed and the system as used for the spot formation measurements will be presented. The TPU approach taken here is the one presented by us in. ${ }^{4}$ Alternatives to this approach exist, like for example. ${ }^{5}$ The pro's and cons of these two methods have been discussed in literature. ${ }^{6}$ 


\subsection{TPU basics}

In TPU, the idea is not to unwrap the phase in space, but to follow the phase difference evolution between reference and object beam, and add all phase increments. As long as the phase change between two successive recordings is between $-\pi$ and $\pi$, the phase obtained by accumulation of all these phase changes will be inherently unwrapped. This directly indicates the weak point in the TPU approach: it can not be used for static phase measurements. The strong point is that it can be used to measure phase changes, and since only the changes are recorded the actual phase distribution is of no importance. This allows for the use of low quality optics.

In order to be able to calculate the phase change two approaches can be followed. The first is to record at each time enough phase stepped images to calculate the phase, followed by a subtraction of the two phase distributions for the two successive recordings. This requires a minimum of three phase stepped images per time. We have chosen for a different approach where the actual phase is never calculated but where only the phase difference is obtained. In our approach two phase stepped images per time suffice.

For each time $t$ two phase stepped images are recorded

$$
\begin{aligned}
I_{0}(t) & =I_{B}+I_{M} \cos (\varphi(t)), \\
I_{\pi / 2}(t) & =I_{B}-I_{M} \sin (\varphi(t)),
\end{aligned}
$$

where $I_{B}$ and $I_{M}$ are, respectively, the background and modulation intensity. The quantity $\varphi(t)$ denotes the phase difference between the object and the reference beam. The object beam is taken to be reflected on the part of the surface being machined while the reference beam is reflected on a part of the surface that is not machined. The phase change between two successive recordings, say recordings at time $t$ and at time $t+T$ can be obtained via

$$
\Delta \varphi(t+T, t)=-\frac{\pi}{2}-2 \arctan \left(\frac{I_{0}(t)-I_{\pi / 2}(t+T)}{I_{\pi / 2}(t)-I_{0}(t+T)}\right),
$$

where the subscript 0 and $\pi / 2$ indicate the phase step between the two interfering beams, the object and reference beam.

All calculated phase changes are added to a so-called accumulator via

$$
\Delta \Phi=\sum_{i=0}^{N} \Delta \varphi(\{i+1\} T, i T) .
$$

From Eqs.(3) and (4) it follows that to calculate the first phase change four interferograms have to be recorded. For each successive phase change only two new interferograms are required, that will be combined with two previous recordings to obtain the phase change information.

The TPU approach has recently also been used for non destructive defect detection. ${ }^{7}$ In that case a dedicated dual image, single camera system was created to capture the two buckets simultaneously.

\subsection{Measurement Setup}

For the monitoring of spot formation during fluid jet polishing a rigid system had to be constructed that was suited for the use in a wet and vibrating environment. In this section the setup will be discussed. First the idea will be highlighted on basis of Fig. 2. As can be seen in Fig. 2, the probing light beams enter the surface from beneath. In this way the light is reflected under total internal reflection conditions, such that only the surface shape is measured without influence of the slurry on top of the surface. The incoming light beam is collimated and has the function to illuminate the spot being processed and a reference area. The machined spot and the reference spot are then, after combination, imaged onto the two CCD camera's in the system where they interfere thus providing us the two phase stepped images required for the 2-bucket TPU algorithm. The indicated cover plate prevents the optics to get wet by the slurry. For more insight in the layout also a top view is included, see Fig. 3. From Fig. TopView it can be seen that the laser beam, after collimation, is split into two beams by a beam splitter (BS). One beam is reflected, from within, by the spot being machined while the other is reflected on a piece of the workpiece that is not machined as reference. One of the beams 


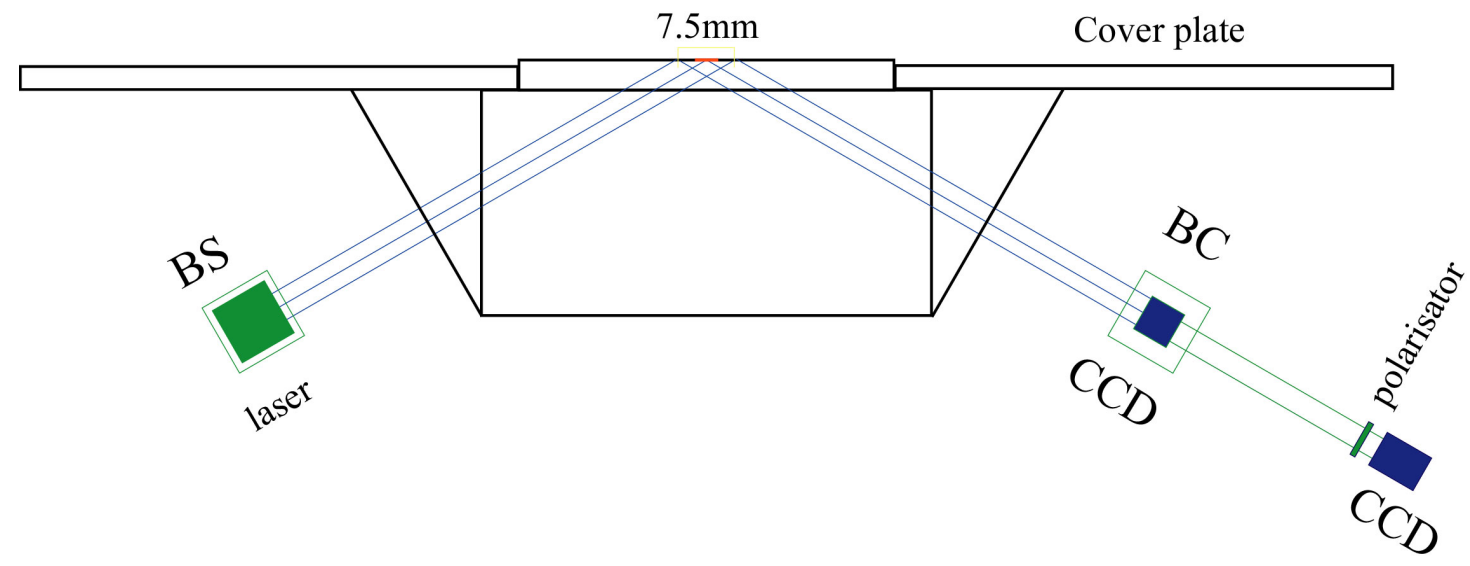

Figure 2. Side view of the measurement setup.

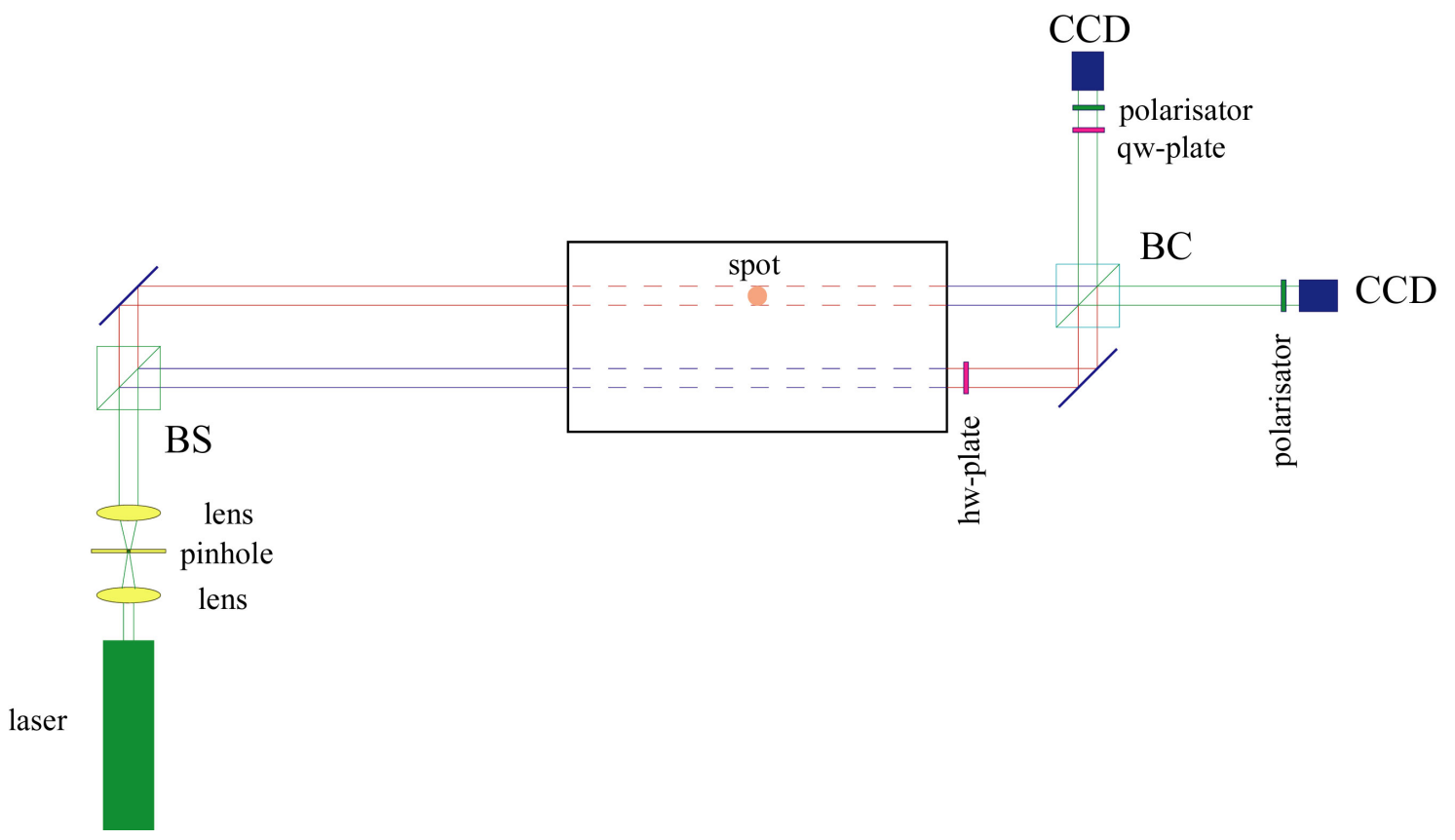

Figure 3. Top view of the measurement setup.

is transmitted through a half wave plate (hw-plate) to change the polarization direction over 90 degrees. A polarizing beam splitting cube (BC) is used to combine the two beams. Before the beams impinge on the CCD camera a polarization plate is inserted to obtain interference. In one of the paths to the CCD camera's a quarter wave plate (qw-plate) is inserted to provide an additional $\pi / 2$ phase step. The lenses to image the measured spots onto the CCD camera's are omitted from this schematic drawing.

From Figs. 2 and 4 it can be seen that the sample on which the spot is being created is placed on a special holder. The gap between sample and holder is filled with an index matching fluid. The facets of the holder are slanted to obtain perpendicular incidence on the entrance and exit while the beams propagate within the material under an angle exceeding the angle of total internal reflection with respect to the surface being processed.

In Fig. 4 the shielding of the optical components for the slurry can be seen. The object being processed can 


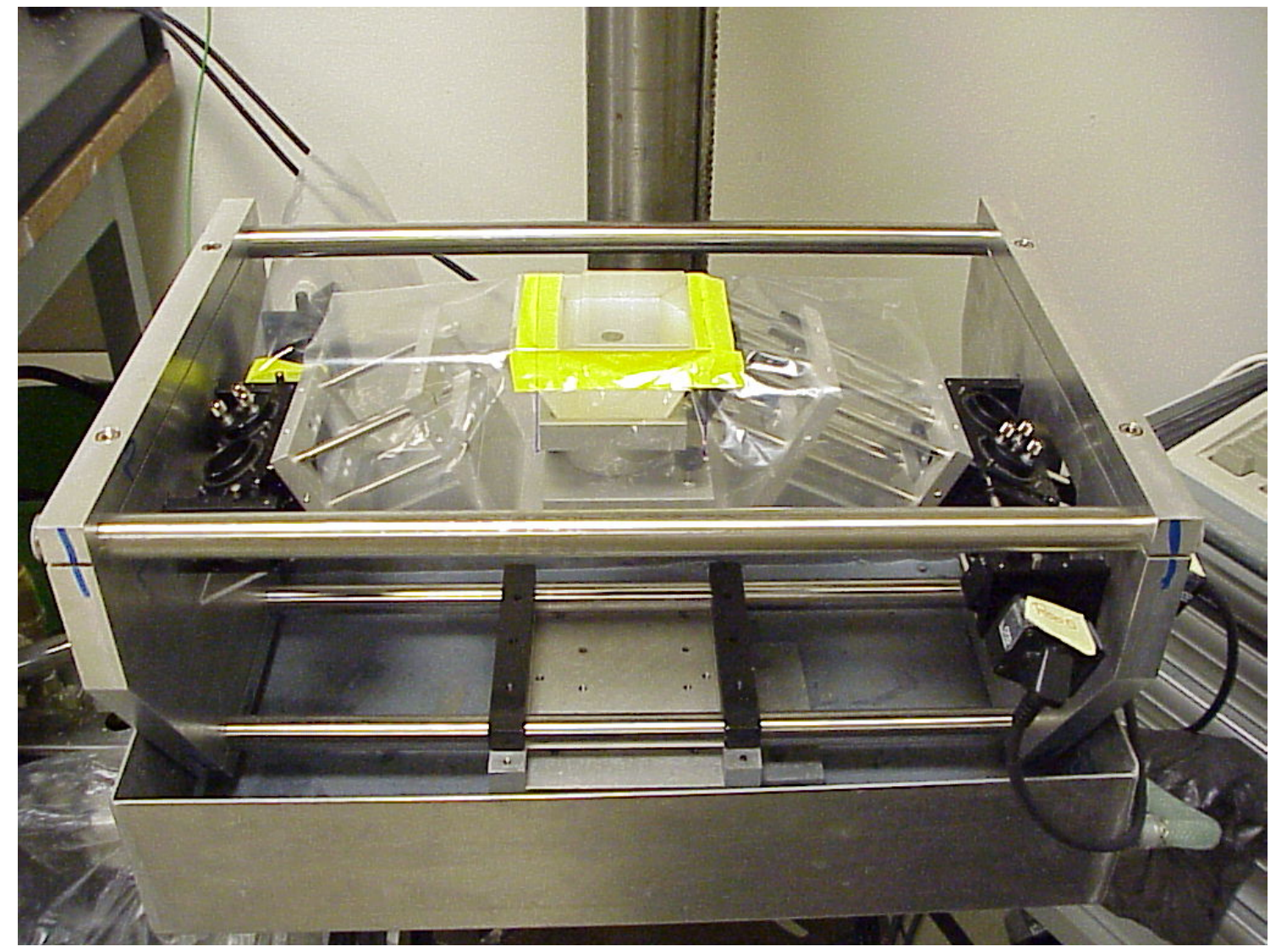

Figure 4. Photograph of the constructed measurement system.

be seen on top of the holder. The whole measurement system was designed to fit into the slurry collector of the FJP system. The CCD camera's can be seen on the right hand side of the image. The laser used for this setup was a $5 \mu W$ HeNe laser $(\lambda=632.8 \mathrm{~nm})$ and the output of this laser is guided toward the setup via an optical single mode fiber. The light intensity inside the system was lowered to levels acceptable to the CCD camera's by changing the coupling efficiency of the laser beam into the fiber.

\section{RESULTS AND DISCUSSION}

In this paper a single experiment will be presented. The idea behind this setup is to investigate the spots as created by the FJP process while the nozzle remains stationary with respect to the surface. The outcome of the measurements will be a test for the derived model of the spot formation as presented in literature. ${ }^{3}$

For the presented test measurement we set the FJP angle to about $45^{\circ}$ and used a standard slurry $(10 \% \# 800$ $\mathrm{SiC}$ ). We nozzled for three minutes and arrived at a maximum depth of $3 \mu \mathrm{m}$ in the deepest part of the produced hollow. During the material removal we recorded the interferograms, two for each time $t$. One with a zero phase step $\left(I_{0}(t)\right)$, the other with a $\pi / 2$ phase step $\left(I_{\pi / 2}(t)\right)$. The images pertaining to the zero phase step are shown in Fig. 5. The first image is shown in the top left hand corner, from there the images are ordered from left to right with time of recording, and then from top to bottom. Only every fifth image is shown in order to reduce the number of images.

The program used for image capture and data analysis was written in LabView. Although this is a versatile programming environment the resulting programs are relatively slow. The system could capture one set of two phase stepped images per five seconds. Owing to the fact that the FJP system has under the here set conditions 


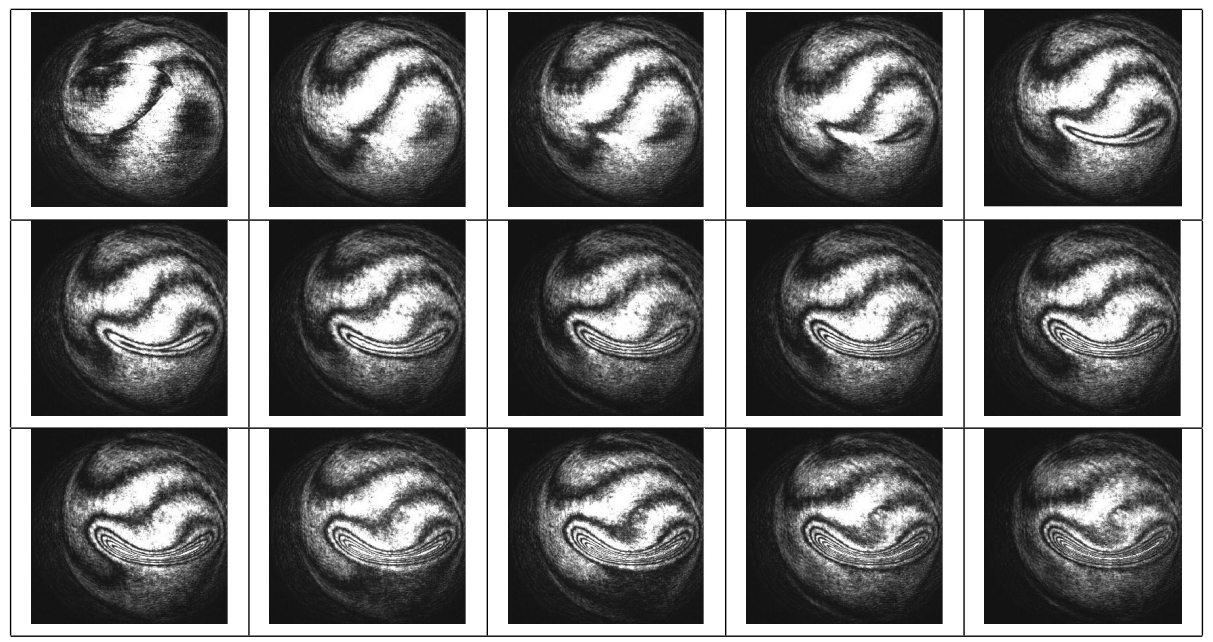

Figure 5. Obtained interferograms as a function of processing time. The obtained depth in the deepest part is about $3 \mu \mathrm{m}$.

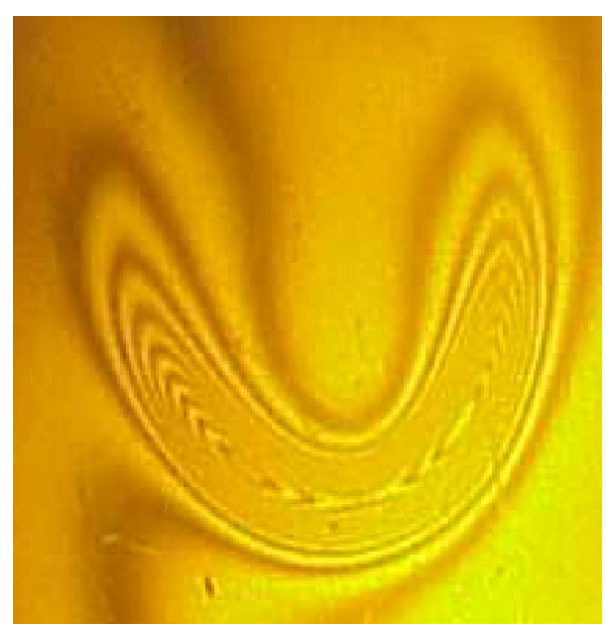

Figure 6. Verification of the final result shown in Fig. 5. The Newton fringes in sodium light are shown as obtained by placing the work piece on a flat reference sample.

a low, and thereby accurate, removal rate, the phase changes were never outside the allowed range pointed out before.

The first image is obtained from four images that were recorded prior to the processing. On this image the influence of a water droplet can be seen. The fact that this effect can only be seen on the first image shows that a wet layer causes no problem. The reason that we can see the droplet on the surface, while we are reflecting within the angle of total internal reflection, can be explained by assuming that the droplet is thin enough to get reflection from the top interface. The light that is present within the droplet is the evanescent tail. If the droplet, or layer, is thin enough for this tail to be twice the thickness then effect of the top surface can be seen in the reflected light beam.

As a function of time the number of fringes due to the nozzled spot increases until a clear 'smiley' is formed. As check on the fidelity of the obtained interferograms an image obtained by a simple test glass arrangement (Newton fringes) is also shown, see Fig. 6.

The horse shoe, or 'smiley' shaped spot in Fig. 6 is elongated with respect to the spots in Fig. 5. This can readily be explained by the fact that the in-process measurement are performed under a large angle while the 
Newton fringes were recorded perpendicular to the surface.

The depth obtained from a simple fringe counting approach in the Newton fringes (Fig. 6) was $\approx 3 \mu m$ while the depth calculated from the TPU data resulted in $2.8 \pm 0.1 \mu \mathrm{m}$. The error margin in this value originates from the noise level in the height data. Although the system was designed to be as rigid as possible it was found that the fringes outside the machined area shifted as a function of time. This indicates that the sample was not rigidly fixed to the holder. Since the sample was only 'glued' with an index matching fluid it very well could be the case that the sample drifts away by the force of the slurry jet impinging on it. The shift in fringe positions can be seen in the interferograms of Fig. 5.

To convert the phase change to a material removal the angle of incidence has to be taken into account, as well as the wavelength used and the refractive index of the glass being processed. The phase change shows, due to fact that we measure in reflection, a double dependence on the spot depth. The change in path length $L$ for the beam reflected on the spot, with respect to the reference beam is found to be

$$
L=2 d \cos (\alpha),
$$

where $d$ is the change in spot depth, and $\alpha$ is the angle of incidence of the probing beam with respect to the surface normal. The phase difference between object and reference beams, due to this path length change $L$, is

$$
\varphi=\frac{2 \pi}{\lambda} L,
$$

in which $\lambda$ is the wavelength of the light used. The phase difference is invariant with the material, being it the sample being processed, the glass of the holder or the air where the beam propagates through near the camera's. The effect is that the apparent height change measured is larger than the actual height change by a factor equal to the refractive index of the material being machined. This can be visualized by remembering that the wavelength inside a material gets shorter due to the higher refractive index of refractive index. This means that the phase difference is equal to

$$
\varphi=\frac{2 \pi n(\lambda)}{\lambda} L .
$$

The removed spot depth $\Delta d$ is obtained via a combination of the above given expressions

$$
\Delta d=\frac{\Delta \varphi \lambda}{4 \pi n \cos (\alpha)},
$$

where $\Delta \varphi$ is the measured change in phase difference between object and reference beam. From Eq.(8) it follows directly that for accurate measurements the refractive index of the material being FJP'ed upon has to be known precisely, as well as the angle of incidence $\alpha$ inside the material.

The value of $d=3 \mu \mathrm{m}$ corresponds to a phase change between object and reference of about $14 \pi$ (as follows directly from Eq.(8)), which equals seven fringes. In the bottom right hand image of Fig. 5 this is the number of fringes that are added with respect to the image shown in the top left hand side corner. This visual approach yields the correct depth. The automatic system is less accurate due to a shift of the fringes with time.

The final limiting factor in terms of maximum height that can be measured by this monitoring setup is the fringe density. The interferograms that are recorded should contain fringes that can be resolved by the camera's. The minimum number of CCD pixels per fringe should be two. For very accurate measurements a larger number, say about ten, should be used.

The experimental issues were: slurry on the surface and vibrations. These were tackled by measuring from within the sample under total internal reflection conditions and by reflecting both the object and the reference beam on the sample being processed. From the shown interferograms it follows that the created setup works satisfactory. The thing to improve upon is the way in which the sample is fixed inside the setup. It is believed that the sample moved due to the forces of the slurry jet. The alignment of the measured spot with respect to the machined spot will have to be made more flexible. 
The system as used for the discussed experiment will be improved upon in the near future and will be used to monitor the spot shapes and removal during production. The footprint for a large number of nozzle types and shapes will be investigated and the outcome of the measurements will be used to arrive at an accurate experimental knowledge of the FJP process allowing it to be used for high precision, optical components production.

\section{CONCLUSIONS}

The presented results proof that the temporal phase unwrapping method can be used to monitor, in-process, the spot formation as encountered in fluid jet polishing. Although the sample on which we used the FJP system was not rigidly connected to its holder we were still able to measure a relatively accurate footprint of the used nozzle at the given angle which showed excellent agreement with our theory. The depth value is less accurate which is assumed to be due to the drift of the sample caused by the forces induced on it by the slurry jet.

\section{REFERENCES}

1. R. M. van der Bijl, O. W. Fänhnle, and H. van Brug, "Subsurface damage measurements as a tool for process monitoring," in Proceedings of the ASPE 1999 annual meeting, 20, pp. 606 - 609, 1999.

2. O. W. Fähnle, H. van Brug, and H. J. Frankena, "Fluid jet polishing of optical surfaces," Appl. Opt. 37(28), pp. $6771-6773,1998$.

3. S. M. Booij, H. van Brug, and O. W. Fähnle, "A mathematical model for machining spot in Fluid Jet Polishing," in Optical Fabrication and Testing, A. Lindquist, ed., Technical Digest Series, pp. 70 - 72, OSA, 2000.

4. H. van Brug, "Temporal phase unwrapping and its application in shearography systems," Appl. Opt. 37(28), pp. $6701-6706,1998$.

5. J. M. Huntley and H. Saldner, "Temporal phase-unwrapping algorithm for automated interferogram analysis," Applied Optics 32, pp. 3047 -3052, 1993.

6. H. van Brug and P. A. A. M. Somers, "Temporal phase unwrapping with two or four images per time frame: a comparison," in Interferometry '99: Techniques and Technologies, International conference on optical metrology, M. Kujawinska and M. Takeda, eds., 3744, pp. 358 - 365, SPIE, 1999.

7. P. A. A. M. Somers and H. van Brug, "A single camera, dual image real-time-phase-stepped shearing speckle interferometer," in Fringe 2001, W. Osten and W. Jüptner, eds., pp. 573 -580, Elsevier, 2001. 